\title{
EVALUATIONS OF CERTAIN CATALAN-HANKEL PFAFFIANS VIA CLASSICAL SKEW ORTHOGONAL POLYNOMIALS
}

\author{
BO-JIAN SHEN, SHI-HAO LI, AND GUO-FU YU
}

\begin{abstract}
This paper is to evaluate certain Catalan-Hankel Pfaffians by the theory of skew orthogonal polynomials. Due to different kinds of hypergeometric orthogonal polynomials underlying the Askey scheme, we explicitly construct the classical skew orthogonal polynomials and then give different examples of Catalan-Hankel Pfaffians with continuous and $q$-moment sequences.
\end{abstract}

\section{INTRODUCTION}

Hankel determinants as a specific determinant has attracted much attention from researchers in many different subjects (see, for example $[5,12,29]$ and references therein). It is well known that if $\left\{\mu_{m}\right\}_{m \geq 0}$ is the moment sequence taking the form

$$
\mu_{n}=\int_{\mathbb{R}} x^{n} \omega(x) d x
$$

then the Hankel determinant $\operatorname{det}\left(\mu_{i+j}\right)_{i, j=0}^{n-1}$ has a nice integral formula due to the Andreiéf formula $[14]$

$$
\operatorname{det}\left(\mu_{i+j}\right)_{i, j=0}^{n-1}=\frac{1}{n !} \int_{\mathbb{R}^{n}} \prod_{1 \leq i<j \leq n}\left|x_{i}-x_{j}\right|^{2} \prod_{i=1}^{n} \omega\left(x_{i}\right) d x_{i} .
$$

Moreover, if $\omega(x)$ is a classical weight, e.g. the Gaussian weight, Laguerre weight, Jacobi weight and circular Jacobi weight, then Hankel determinant with classical moments can be explicitly evaluated due to the Selberg integral [13, Chapter 4]. Moreover, $q$-versions of Selberg integrals which relate to the moment sequence with discrete measure play important roles in modern mathematical physics as well [17].

In recent years, the Pfaffian version of Hankel determinant, called the Catalan-Hankel Pfaffian, was proposed due to its potential applications in the theory of combinatorics [19]. The Pfaffian version of Andreiéf formula, which is called the de Bruijn formula, has been applied into the evaluation of certain Catalan-Hankel Pfaffian with the help of the Selberg integral and its $q$-version $[19,20]$. Catalan-Hankel Pfaffian has the form

$$
\operatorname{Pf}\left[(j-i) \mu_{i+j-1}\right]_{i, j=0}^{2 N-1}
$$

and its $q$-version

$$
\operatorname{Pf}\left[\left([j]_{q}-[i]_{q}\right) \mu_{i+j-1}\right]_{i, j=0}^{2 N-1}, \quad[j]_{q}=\frac{1-q^{j}}{1-q}
$$

2010 Mathematics Subject Classification. 15A15, 33E20.

Key words and phrases. Catalan-Hankel Pfaffian; Classical orthogonal polynomials; Skew orthogonal polynomials. 
where $\left\{\mu_{n}\right\}_{n \geq 0}$ is a moment sequence related to discrete $q$-measure. The specific little $q$-Jacobi case were considered in [19] and some recent developments like the Al-Salam \& Carlitz I case was given in [20].

Regarding evaluations of ( $q$-)Catalan-Hankel Pfaffian, one application is to give formula to the weighted enumeration of some specific plane partitions. For example, in [19], the authors considered the little $q$-Jacobi case and enumerated a special family of shifted reverse plane partitions with weights that resemble the weight in the inner product of little $q$-Jacobi polynomials. The importance of these evaluations lies in the random matrix theory as well. In an earlier work of Mehta and Wang [25], they gave an evaluation of the Hankel Pfaffian

$$
\operatorname{Pf}[(j-i) \Gamma(i+j+b)]_{i, j=0}^{2 N-1},
$$

which was related to the skew orthogonal Laguerre polynomials given in [1]. Moreover, the shifted Catalan-Hankel Pfaffian

$$
\operatorname{Pf}\left[(j-i) \mu_{i+j+r}\right]_{i, j=0}^{2 N-1} \quad \text { or } \quad \operatorname{Pf}\left[\left([j]_{q}-[i]_{q}\right) \mu_{i+j+r}\right]_{i, j=0}^{2 N-1}
$$

is closely related to the adjacent families of skew-orthogonal polynomials considered in [23]. These evaluations may give more hints in exploring novel examples in integrable systems and random matrices.

As mentioned, one way to evaluate these Pfaffians is based on the de Bruijn formula and Selberg integral $[19,20]$. In this paper, we will investigate another way, namely by using the theory of classical (q-)skew orthogonal polynomials, to make these evaluations and demonstrate the effectiveness - by simply considering different classsical weights underlying Askey scheme, we can get different examples of $(q-)$ Catalan-Hankel Pfaffians. Since the Hankel determinant is closely related to the theory of classical orthogonal polynomials, then if we know normalisation constants of these classical orthogonal polynomials, the evaluations of Hankel determinant can be made. It is natural to ask whether we can apply the theory of classical ( $q$-)skew orthogonal polynomials into evaluations of the certain ( $q$-)Catalan-Hankel Pfaffians. The answer is affirmative. If we know the normalisation factors of skew orthogonal polynomials, then these Pfaffians can be explicitly given by those normalisation factors. Due to different expressions in discrete and continuous cases (c.f. (1.1) and (1.2)), we discuss them separately. Continuous cases including Hermite-type, Laguerretype and Jacobi-type skew orthogonal polynomials were firstly constructed by Adler et al [1] and we give a brief review in section 2. Some evaluations of Catalan-Hankel Pfaffians related to these weights including formula (1.3) will be demonstrated. Moreover, we consider a Catalan-Hankel Pfaffian with Cauchy weight in that part. Evaluations of $q$-Catalan-Hankel Pfaffians will be based on the theory of classical $q$-skew orthogonal polynomials. With the help of discrete Pearson relation, we construct different examples of classical $(q$-)skew orthogonal polynomials, thus obtaining different kinds of skew orthogonal polynomials and normalisation factors with respect to different classical $q$-weights, which enlarge the results given by [16, 20].

\section{Continuous measure}

Let's consider the skew inner product related to (1.1)

$$
\langle\phi(x), \psi(x)\rangle_{4, \omega}=\frac{1}{2} \int_{\mathbb{R}}\left[\phi(x) \psi^{\prime}(x)-\phi^{\prime}(x) \psi(x)\right] \omega(x) d x,
$$


then the skew moments are given by

$$
m_{i, j}:=\left\langle x^{i}, x^{j}\right\rangle_{4, \omega}=\frac{1}{2}(j-i) \int_{\mathbb{R}} x^{i+j-1} \omega(x) d x .
$$

The skew LU decomposition (or so-called skew Borel decomposition [2]) of the moment matrix $\left(m_{i, j}\right)_{i, j \in \mathbb{N}}$ could give rise to the monic skew orthogonal polynomials $\left\{Q_{j}(x)\right\}_{j \in \mathbb{N}}$ satisfying the skew orthogonal relation

$$
\left\langle Q_{2 n}(x), Q_{2 m+1}(x)\right\rangle_{4, \omega}=u_{n} \delta_{n, m}, \quad\left\langle Q_{2 n}(x), Q_{2 m}(x)\right\rangle_{4, \omega}=\left\langle Q_{2 n+1}(x), Q_{2 m+1}(x)\right\rangle_{4, \omega}=0
$$

for certain $u_{n}>0$. Moreover, these skew orthogonal polynomials have the following Pfaffian expressions [8]

$$
Q_{2 n}(x)=\frac{1}{\tau_{2 n}} \operatorname{Pf}(0, \cdots, 2 n, x), \quad Q_{2 n+1}(x)=\frac{1}{\tau_{2 n}} \operatorname{Pf}(0, \cdots, 2 n-1,2 n+1, x)
$$

with $\tau_{2 n}=\operatorname{Pf}(0, \cdots, 2 n-1)$ and the Pfaffian elements are given by $\operatorname{Pf}(i, j)=m_{i, j}$ and $\operatorname{Pf}(i, x)=x^{i}$. By putting $m_{i, j}$ into the expression of $\tau_{2 n}$, we have

$$
\tau_{2 n}=\frac{1}{2^{n}} \operatorname{Pf}\left[(j-i) \mu_{i+j-1}\right]_{i, j=0}^{2 n-1}, \quad \mu_{n}=\int_{\mathbb{R}} x^{n} \omega(x) d x .
$$

Interestingly, $u_{n}$ in the skew orthogonal condition (2.2) is the ratio $\tau_{2 n+2} / \tau_{2 n}$, and therefore, if we can explicitly compute $\left\{u_{n}\right\}_{n \in \mathbb{N}}$ in the skew orthogonal condition, then we directly have $\tau_{2 N}=$ $\prod_{i=0}^{N-1} u_{i}$. In fact, normalisation factors $\left\{u_{n}\right\}_{n \in \mathbb{N}}$ can be explicitly computed when $\left\{Q_{n}(x)\right\}_{n \in \mathbb{N}}$ are classical skew orthogonal polynomials. For details, please refer to [1] and we give a brief review here.

Starting from the inner product

$$
\langle\phi(x), \psi(x)\rangle_{2, \rho}=\int_{\mathbb{R}} \phi(x) \psi(x) \rho(x) d x,
$$

one can construct a family of monic orthogonal polynomials $\left\{p_{j}(x)\right\}_{j \in \mathbb{N}}$ satisfying the orthogonal relation

$$
\left\langle p_{j}(x), p_{k}(x)\right\rangle_{2, \rho}=h_{j} \delta_{j, k}
$$

We call these orthogonal polynomials classical if the weight function $\rho(x)$ satisfies the following Pearson equation

$$
\frac{\rho^{\prime}(x)}{\rho(x)}=-\frac{g(x)}{f(x)} \quad \text { with deg } f(x) \leq 2 \text { and } \operatorname{deg} g(x) \leq 1 .
$$

From the relation, one can construct an operator

$$
\mathcal{A}=f \partial_{x}+\frac{f^{\prime}-g}{2}
$$

such that

$$
\langle\phi(x), \mathcal{A} \psi(x)\rangle_{2, \rho}=\langle\phi(x), \psi(x)\rangle_{4, \omega}, \quad \omega(x)=\rho(x) f(x),
$$

where the skew inner product $\langle\cdot, \cdot\rangle_{4, \omega}$ was given in (2.1). This is the key formula to establish the relation between classical orthogonal polynomials and classical skew orthogonal polynomials. Moreover, the normalisation factor $u_{n}$ could be computed via

$$
\mathcal{A} p_{k}(x)=-\frac{c_{k}}{h_{k+1}} p_{k+1}(x)+\frac{c_{k-1}}{h_{k-1}} p_{k-1}(x), \quad u_{n}=c_{2 n}
$$


with $h_{k}$ the normalisation factor of orthogonal polynomials. Therefore, to obtain normalisation factors of skew orthogonal polynomials, our attention should be paid to the computation of $c_{k}$ in the above equation. The fastest method to compute $c_{k}$ is to compare the coefficients of $x^{k+1}$ on both sides and the following are the examples of the continuous classical weights including Hermite, Laguerre, Jacobi and Cauchy weights.

From weight functions

$$
\rho(x)= \begin{cases}e^{-x^{2}}, & \text { Hermite } \\ x^{a} e^{-x}, & \text { Laguerre } \\ x^{a}(1-x)^{b}, & \text { Jacobi } \\ \left(1+x^{2}\right)^{-a}, & \text { Cauchy }\end{cases}
$$

one can obtain the Pearson pair

$$
(f, g)= \begin{cases}(1,2 x), & \text { Hermite } \\ (x, x-a), & \text { Laguerre } \\ (x(1-x),(a+b) x-a), & \text { Jacobi } \\ \left(1+x^{2}, 2 a x\right), & \text { Cauchy }\end{cases}
$$

This table was given by [13, eq. (5.58)] and it should be remarked that we use the weight function of Jacobi as $x^{a}(1-x)^{b}$ supported in $[0,1]$ to make the moments easily written down. There are two quantities obtained from this table. One is the Hankel sequences given in (2.3). From $\omega(x)=f(x) \rho(x)$, one knows that the weights of classical skew orthogonal polynomials are

$$
\omega^{(H)}(x)=e^{-x^{2}}, \quad \omega^{(L)}(x)=x^{a+1} e^{-x}, \quad \omega^{(J)}(x)=x^{a+1}(1-x)^{b+1}, \quad \omega^{(C)}(x)=\left(1+x^{2}\right)^{-a+1} .
$$

Therefore, we have the following moments form

$$
\begin{array}{ll}
\mu_{n}^{(H)}=\frac{1+(-1)^{n}}{2} \Gamma\left(\frac{n+1}{2}\right), & \mu_{n}^{(L)}=\Gamma(n+a+2), \\
\mu_{n}^{(J)}=\frac{\Gamma(n+a+2) \Gamma(b+2)}{\Gamma(n+a+b+4)}, & \mu_{n}^{(C)}=\frac{1+(-1)^{n}}{2} \frac{\Gamma((n+1) / 2) \Gamma(a-2-(n+1) / 2)}{\Gamma(a-1)} .
\end{array}
$$

On the other hand, we can obtain

$$
c_{k}= \begin{cases}h_{k+1}^{(H)}, & \text { Hermite } \\ h_{k+1}^{(L)} / 2, & \text { Laguerre } \\ (k+1+(a+b) / 2) h_{k+1}^{(J)}, & \text { Jacobi } \\ (a-1-k) h_{k+1}^{(C)}, & \text { Cauchy }\end{cases}
$$

where $\left\{h_{k}\right\}_{k \in \mathbb{N}}$ are normalisation factors with respect to different weights [13, Chap. 5]

$$
\begin{aligned}
& h_{k}^{(H)}=\pi^{1 / 2} 2^{-k} k !, \quad h_{k}^{(L)}=\Gamma(k+1) \Gamma(a+k+1), \\
& h_{k}^{(J)}=2^{a+b+1+2 k} \frac{\Gamma(k+1) \Gamma(a+b+1+k) \Gamma(a+1+k) \Gamma(b+1+k)}{\Gamma(a+b+2 k+1) \Gamma(a+b+2 k+2)}, \\
& h_{k}^{(C)}=\pi 2^{2 k+2 a+2} \frac{\Gamma(k+1) \Gamma(-2 k-2 a) \Gamma(-2 a-2 k-1)}{\Gamma(-2 a-k)(\Gamma(-a-k))^{2}} .
\end{aligned}
$$

By using above results, we could state the following proposition. 
Proposition 2.1. We have the following evaluations of certain Catalan-Hankel Pfaffians

$$
\begin{aligned}
& \operatorname{Pf}\left[(j-i) \mu_{i+j-1}^{(H)}\right]_{i, j=0}^{2 N-1}=2^{-N(N-1)}(\sqrt{\pi})^{N} \prod_{i=0}^{N-1} \Gamma(2 i+2), \\
& \operatorname{Pf}\left[(j-i) \mu_{i+j-1}^{(L)}\right]_{i, j=0}^{2 N-1}=\prod_{i=0}^{N-1} \Gamma(2 i+2) \Gamma(2 i+a+2), \\
& \operatorname{Pf}\left[(j-i) \mu_{i+j-1}^{(J)}\right]_{i, j=0}^{2 N-1}=4^{N} \frac{\Gamma(N+(a+b+2) / 4)}{\Gamma((a+b+2) / 4)} \prod_{i=0}^{N-1} \frac{\Gamma(a+2 i+2) \Gamma(b+2 i+2) \Gamma(2 i+3)}{\Gamma(a+b+4 i+3)}, \\
& \operatorname{Pf}\left[(j-i) \mu_{i+j-1}^{(C)}\right]_{i, j=0}^{2 N-1}=(-1)^{N} 2^{2 N^{2}-N(a-3)} \frac{\Gamma(N+(1-a) / 2)}{\Gamma((1-a) / 2)} \prod_{i=0}^{N-1} \frac{\Gamma(2 a-4 i-2) \Gamma(2 i+3)}{(\Gamma(a-2 i-1))^{2}} .
\end{aligned}
$$

Remark 2.2. The second formula is exactly equation (1.3) with the shift $a \rightarrow a-1$.

\section{DisCRETE MEASURE: $q$-CASE}

This part is devoted to the evaluations of $q$-Catalan Hankel Pfaffians given by formula (1.2). The $q$-case corresponds to a special discrete measure distributed on exponential lattices $x(i)=q^{i}$ with $0<q<1$ and $i \in \mathbb{Z}$. By using the definition of Jackson's $q$-integral ${ }^{1}$

$$
\int_{0}^{\infty} f(x) d_{q} x=(1-q) \sum_{s=-\infty}^{\infty} f\left(q^{s}\right) q^{s}
$$

one can define the following inner product

$$
\langle\phi(x), \psi(x)\rangle_{2, \rho}:=\int_{0}^{\infty} \phi(x) \psi(x) \rho(x) d_{q} x=(1-q) \sum_{s \in \mathbb{Z}} \phi\left(q^{s}\right) \psi\left(q^{s}\right) \rho\left(q^{s}\right) q^{s} .
$$

With this inner product, $q$-orthogonal polynomials $\left\{p_{n}(x ; q)\right\}_{n \in \mathbb{N}}$ are defined by the orthogonal relation

$$
\left\langle p_{i}(x ; q), p_{j}(x ; q)\right\rangle_{2, \rho}=h_{n}(q) \delta_{n, m}
$$

with respect to the weight $\rho(x ; q)$. Moreover, if $\rho(x ; q)$ is a classical weight, we call the corresponding orthogonal polynomials the classical $q$-orthogonal polynomials. Classical $q$-orthogonal polynomials include many interesting examples underlying the $q$-Askey scheme. For details, please refer to $[3,21,22]$. One important property of classical $q$-orthogonal polynomials is that the weight function satisfies an analogy of Pearson relation given by Nikiforov and Suslov [26]

$$
\frac{\rho(q x)}{\rho(x)}=\frac{f(x)-q^{-\frac{1}{2}}(1-q) x g(x)}{f(q x)} \quad \text { with } \operatorname{deg} f(x) \leq 2 \text { and } \operatorname{deg} g(x) \leq 1 .
$$

In the following, we will demonstrate how to connect $q$-inner product with $q$-skew inner product.

Let's define a $q$-analogy of the skew inner product (2.1)

$$
\langle\phi(x), \psi(x)\rangle_{4, \omega}=\int_{0}^{\infty}\left[\phi(x) D_{q} \psi(x)-D_{q} \phi(x) \psi(x)\right] \omega(x) d_{q} x
$$

with $q$-difference operator

$$
D_{q} f(x)=\frac{f(x)-f(q x)}{(1-q) x} .
$$

\footnotetext{
${ }^{1}$ The definition of $q$-integral on the interval $[0, \infty)$ is different from the one defined on $[0,1]$, see [21]. However, these two cases can be treated similarly and we just consider the former one here.
} 
Then by defining an operator $\mathcal{A}_{q}[16]$

$$
\mathcal{A}_{q}=q^{-\frac{1}{2}} g(x) T_{q}+q^{-1} f(x) D_{q^{-1}}+f(x) D_{q}, \quad T_{q} f(x)=f(q x),
$$

one can find such a connection formula

$$
\left\langle\phi(x ; q), \mathcal{A}_{q} \psi(x ; q)\right\rangle_{2, \rho}=\langle\phi(x ; q), \psi(x ; q)\rangle_{4, \omega}, \quad \omega(x)=\rho(q x) f(q x) .
$$

On the other hand, from the skew inner product (3.1), one has the following $(q-)$ skew moments

$$
m_{i, j}:=\left([j]_{q}-[i]_{q}\right) \int_{0}^{\infty} x^{i+j-1} \omega(x) d_{q} x, \quad[j]_{q}=\frac{1-q^{j}}{1-q} .
$$

Similarly, it is known that a family of monic skew orthogonal polynomials $\left\{Q_{i}(x ; q)\right\}_{i \in \mathbb{N}}$ could be constructed from those moments if even-order moment matrices $\operatorname{Pf}\left(m_{i, j}\right)_{i, j=0}^{2 n-1} \neq 0$ for all $n \in \mathbb{N}_{+}$ [16]. Furthermore, polynomials $\left\{Q_{i}(x ; q)\right\}_{i \in \mathbb{N}}$ admit the following Pfaffian expressions

$$
\begin{aligned}
& Q_{2 n}(x ; q)=\frac{1}{\tau_{2 n}(q)} \operatorname{Pf}(0, \cdots, 2 n, x), \quad Q_{2 n+1}(x ; q)=\frac{1}{\tau_{2 n}(q)} \operatorname{Pf}(0, \cdots, 2 n-1,2 n+1, x) \\
& \tau_{2 n}(q)=\operatorname{Pf}(0, \cdots, 2 n-1), \quad \operatorname{Pf}(i, j)=m_{i, j}, \quad \operatorname{Pf}(i, x)=x^{i},
\end{aligned}
$$

and they satisfy the following skew orthogonal relations

$$
\begin{aligned}
& \left\langle Q_{2 n}(x ; q), Q_{2 m+1}(x ; q)\right\rangle_{4, \omega}=\frac{\tau_{2 n+2}(q)}{\tau_{2 n}(q)}:=u_{n}(q) \delta_{n, m}, \\
& \left\langle Q_{2 n}(x ; q), Q_{2 m}(x ; q)\right\rangle_{4, \omega}=\left\langle Q_{2 n+1}(x ; q), Q_{2 m+1}(x ; q)\right\rangle_{4, \omega}=0 .
\end{aligned}
$$

Therefore, one knows that

$$
\operatorname{Pf}\left[\left([j]_{q}-[i]_{q}\right) \int_{0}^{\infty} x^{i+j-1} \omega(x) d_{q} x\right]_{i, j=0}^{2 N-1}=\operatorname{Pf}(0, \ldots, 2 N-1)=\prod_{i=0}^{N-1} u_{i}(q) .
$$

Interestingly, there is a method to evaluate the value of $u_{n}(q)$ quickly by taking advantage of $q$-Pearson relation.

As an analogy of equation (2.5), there holds the formula

$$
\mathcal{A}_{q} p_{k}(x ; q)=-\frac{c_{k}(q)}{h_{k+1}(q)} p_{k+1}(x ; q)+\frac{c_{k-1}(q)}{h_{k-1}(q)} p_{k-1}(x ; q)
$$

in the $q$-case due to the property of $\mathcal{A}_{q}\left[16\right.$, eq. (4.28)]. Moreover, the quantity $h_{k}(q)$ is the normalisation factor of the orthogonal relation (3.2) and $c_{k}(q)$ is closely related to $u_{k}(q)$ via the relation $u_{k}(q)=c_{2 k}(q)$. Therefore, it is the point to compute $c_{k}(q)$ from above equation and then the exact value of $q$-Catalan Hankel Pfaffian could be obtained. In the following part, we discuss several different cases including the examples in [19, 20].

3.1. Al-Salam \& Carlitz I case. The first example considered here is the Al-Salam \& Carlitz I case with the weight function

$$
\rho(x ; q)=\left(q x, a^{-1} q x ; q\right)_{\infty}, \quad a<0 .
$$

It is well known that the Al-Salam \& Carlitz polynomials $\left\{U_{n}^{(a)}(x ; q)\right\}_{n \geq 0}$ have the orthogonality

$$
\int_{a}^{1} U_{m}^{(a)}(x ; q) U_{n}^{(a)}(x ; q) \rho(x ; q) d_{q} x=(-a)^{n}(1-q)(q ; q)_{n}\left(q, a, a^{-1} q ; q\right)_{\infty} q^{\left(\begin{array}{c}
n \\
2
\end{array}\right)} \delta_{n, m}:=h_{n} \delta_{n, m},
$$


and canonical moments were given by [27]

$$
\mu_{n}=\int_{a}^{1} x^{n} \rho(x ; q) d_{q} x=(1-q)\left(q, a, a^{-1} q ; q\right)_{\infty} \sum_{i=0}^{n}\left[\begin{array}{c}
n \\
i
\end{array}\right]_{q} a^{i}
$$

Specifically, Al-Salam \& Carlitz I polynomials have $q$-hypergeometric function expressions

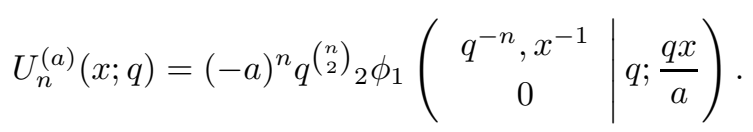

As was shown in [16, Sec. 4.4.1(1)], the Pearson pair in this case is

$$
(f, g)=\left(x^{2}-(1+a) x+a, \frac{q^{1 / 2}}{1-q}(x-(1+a))\right)
$$

and the coefficient $c_{n}$ in (3.8) can be explicitly computed as $c_{n}=-q^{-n} h_{n+1} /(1-q)$. Thus the moment Pfaffian can be written as

$$
\begin{aligned}
\operatorname{Pf}\left(m_{i, j}\right)_{i, j=0}^{2 N-1} & =\prod_{k=0}^{N-1} a^{2 k+1}(q ; q)_{2 k+1} q^{\left(\begin{array}{c}
2 k \\
2
\end{array}\right)}\left(q, a, a^{-1} q ; q\right)_{\infty} \\
& =a^{N^{2}} q^{\frac{1}{6} N(N-1)(4 N-5)}\left(q, a, a^{-1} q ; q\right)_{\infty}^{N} \prod_{k=0}^{N-1}(q ; q)_{2 k+1}
\end{aligned}
$$

where $m_{i, j}=\left([j]_{q}-[i]_{q}\right) \int_{a}^{1} x^{i+j-1} \omega(x ; q) d_{q} x$. Since $\omega(x ; q)=f(q x ; q) \rho(q x ; q)=a \rho(x ; q)$, we have

$$
m_{i, j}=a\left([j]_{q}-[i]_{q}\right) \mu_{i+j-1}=\left(q^{i}-q^{j}\right)\left(q, a, a^{-1} q ; q\right) \sum_{k=0}^{i+j-1}\left[\begin{array}{c}
i+j-1 \\
k
\end{array}\right]_{q} a^{k+1} .
$$

Dividing both sides by $\left[a\left(q, a, a^{-1} q ; q\right)_{\infty}\right]^{N}$ leads to

$$
\operatorname{Pf}\left(\left(q^{i}-q^{j}\right) \sum_{k=0}^{i+j-1}\left[\begin{array}{c}
i+j-1 \\
k
\end{array}\right]_{q} a^{k}\right)_{i, j=0}^{2 N-1}=a^{N(N-1)} q^{\frac{1}{6} N(N-1)(4 N-5)} \prod_{k=0}^{N-1}(q ; q)_{2 k+1} .
$$

Remark 3.1. With $a=-1$, Al-Salam \& Carlitz I polynomials reduce to the q-Hermite I polynomials [22, Sec. 14.28], therefore the above mentioned method can be applied to the q-Hermite I case as well. The moments of q-Hermite I polynomials take the form [27]

$$
\mu_{n}=(1-q)(q,-1,-q ; q)_{\infty} \frac{1+(-1)^{n}}{2}\left(q ; q^{2}\right)_{n / 2}
$$

Thus we have the following evaluation of q-Catalan-Hankel Pfaffian

$$
\operatorname{Pf}\left(\left(q^{i}-q^{j}\right) \frac{1+(-1)^{i+j-1}}{2}\left(q ; q^{2}\right) \frac{i+j-1}{2}\right)_{i, j=0}^{2 N-1}=(-1)^{N} q^{\frac{1}{6} N(N-1)(4 N-5)} \prod_{k=0}^{N-1}(q ; q)_{2 k+1} .
$$

3.2. Stieltjes-Wigert case. The Stieltjes-Wigert polynomials are well studied in random matrix theory of the so-called Stieltjes-Wigert ensemble, which firstly appeared in the study of nonintersecting Brownian walkers, and subsequently in quantum many body systems etc.. For a detailed review, please refer to [15] and references therein. The weight of Stieltjes-Wigert polynomials was first given by Stieltjes as an example of indeterminate moment problems [28] and further 
studied by Wigert [30]. Usually there are several different expressions for the Stieltjes-Wigert's weight function [11]. The original one corresponding to a continuous measure is

$$
w(x)=\frac{1}{\sqrt{\pi}} k x^{-k^{2} \log x}, \quad x>0
$$

with the moment $\mu_{n}=\int_{0}^{\infty} x^{n} w(x) d x=e^{(n+1)^{2} / 4 k^{2}}$. If we set $q=e^{-1 / 2 k^{2}}$, then $\mu_{n}$ can be written as $q^{-(n+1)^{2} / 2}$. With this measure, Wigert found the following expression for StieltjesWigert polynomials [30]

$$
P_{n}(x)=(-1)^{n} \frac{q^{n / 2+1 / 4}}{\sqrt{(q ; q)_{n}}} \sum_{k=0}^{n}\left[\begin{array}{l}
n \\
k
\end{array}\right]_{q}(-1)^{k} q^{k^{2}+k / 2} x^{k},
$$

with orthogonality

$$
\int_{0}^{\infty} P_{n}(x) P_{m}(x) w(x) d x=\delta_{m n} .
$$

In [9], Chihara proposed a discrete weight on the exponential lattices admitting the form

$$
\xi(x)=\left\{\begin{array}{ll}
\frac{1}{\sqrt{q} M} q^{n+n^{2} / 2} & , x=q^{n} \\
0 & , x \neq q^{n}
\end{array} \quad, \quad M=\left(-q \sqrt{q},-q^{-1 / 2}, q ; q\right)_{\infty}, \quad n \in \mathbb{Z} .\right.
$$

Then the corresponding inner product becomes

$$
\left\langle p_{n}(x), p_{m}(x)\right\rangle_{2, \xi}=\frac{1}{\sqrt{q} M} \sum_{k=-\infty}^{\infty} p_{n}\left(q^{k}\right) p_{m}\left(q^{k}\right) q^{k+k^{2} / 2}
$$

We can prove that this discrete weight is equivalent to the continuous one by showing that they have same moments. For completeness, we give a short proof to this fact.

One can easily check that

$$
\xi(q x)=q^{\frac{3}{2}} x \xi(x), \quad x \in \mathbb{R}
$$

and therefore obtain a recurrence relation for the moments $\left\{\mu_{n}\right\}_{n \in \mathbb{N}}$

$$
\begin{aligned}
\mu_{n}:= & \left\langle x^{n}, 1\right\rangle_{2, \xi}=\sum_{k=-\infty}^{\infty} q^{n k} \xi\left(q^{k}\right)=\sum_{k=-\infty}^{\infty} q^{n(k+1)} \xi\left(q^{k+1}\right) \\
& =q^{n+\frac{3}{2}} \sum_{k=-\infty}^{\infty} q^{(n+1) k} \xi\left(q^{k}\right)=q^{n+\frac{3}{2}} \mu_{n+1} .
\end{aligned}
$$

As a result, we have $\mu_{n}=q^{-n^{2} / 2-n} \mu_{0}$. By making use of the Jacobi triple product identity [18, Thm 352]

$$
\sum_{n=-\infty}^{\infty}(-1)^{n} q^{\left(\begin{array}{c}
n \\
2
\end{array}\right)} x^{n}=(x, q / x, q ; q)_{\infty}
$$

$\mu_{0}$ can be directly computed as

$$
\mu_{0}=\frac{1}{\sqrt{q} M} \sum_{k=-\infty}^{\infty} q^{k+k^{2} / 2}=\frac{1}{\sqrt{q}}
$$

Thus $\mu_{n}=q^{-\frac{(n+1)^{2}}{2}}$, which is the same as that of the continuous measure. 
For the present need, we would consider the discrete measure. Denote

$$
\rho(x ; q)=\frac{1}{\sqrt{q} M} x^{\frac{\ln x}{2 \ln q}}
$$

to be the weight function and define

$$
p_{n}(x)=\frac{\sqrt{(q ; q)_{n}}}{q^{n^{2}+n+\frac{1}{4}}} P_{n}(x)
$$

to be the monic Stieltjes-Wigert polynomials. We can rewrite the orthogonality in terms of $\rho(x)$ and $\left\{p_{n}(x)\right\}_{n \geq 0}$ as

$$
\int_{0}^{\infty} p_{n}(x) p_{m}(x) \rho(x) d_{q} x=(1-q) \frac{(q ; q)_{n}}{q^{2 n^{2}+2 n+\frac{1}{2}}} \delta_{m n}:=h_{n} \delta_{n, m} .
$$

By assuming $f(x)=x$ and solving the Pearson equation $(3.3)$, we get $g(x)=\left(q^{2} x-q^{1 / 2}\right) /(q-1)$ which leads to

$$
c_{n}(q)=\frac{q^{n+\frac{3}{2}}}{1-q} h_{n+1} .
$$

As a consequence, we have the following evaluation of the moment Pfaffian

$$
\operatorname{Pf}\left(m_{i, j}\right)_{i, j=0}^{2 N-1}=q^{-\frac{1}{6} N(N+1)(8 N-5)} \prod_{k=0}^{N-1}(q ; q)_{2 k+1} .
$$

On the other hand, we can compute the skew moments (3.7) as a scalar product of canonical moments $\mu_{n}$

$$
\begin{aligned}
m_{i, j} & =\left([j]_{q}-[i]_{q}\right) \int_{0}^{\infty} x^{i+j-1} \omega(x ; q) d_{q} x=\left([j]_{q}-[i]_{q}\right) \int_{0}^{\infty} x^{i+j+1} q^{\frac{3}{2}} \rho(x ; q) d_{q} x \\
& =\left([j]_{q}-[i]_{q}\right) q^{3 / 2} \mu_{i+j+1}=\left([j]_{q}-[i]_{q}\right) q^{-\left[(i+j+2)^{2}-3\right] / 2} .
\end{aligned}
$$

By combining above results, we have the evaluation

$$
\operatorname{Pf}\left(\left([j]_{q}-[i]_{q}\right) q^{-(i+j+2)^{2} / 2}\right)_{i, j=0}^{2 N-1}=q^{-\frac{1}{6} N(2 N+1)(8 N-1)} \prod_{k=0}^{N-1}(q ; q)_{2 k+1} .
$$

3.3. Little $q$-Jacobi case. Little $q$-Jacobi polynomials are important in many mathematical fields such as polynomials theory [22] and quantum group [24]. These polynomials have the following series form (c.f. [24, eq. (2.21)])

$$
p_{n}^{(\alpha, \beta)}(z ; q)=\sum_{r \geq 0} \frac{\left(q^{-n} ; q\right)_{r}\left(q^{\alpha+\beta+n+1} ; q\right)_{r}}{(q ; q)_{r}\left(q^{\alpha+1} ; q\right)_{r}}(q z)^{r}
$$

and they obey the following orthogonal relation [24, Prop. 3.9]

$$
\int_{0}^{1} p_{m}^{(\alpha, \beta)}(z ; q) p_{n}^{(\alpha, \beta)}(z ; q) z^{\alpha}(q z ; q)_{\beta} d_{q} z=\delta_{n, m} q^{(\alpha+1) n} \frac{(1-q)(q ; q)_{\alpha}^{2}(q ; q)_{\beta+n}(q ; q)_{n}}{\left(1-q^{\alpha+\beta+2 n+1}\right)(q ; q)_{\alpha+n}(q ; q)_{\alpha+\beta+n}} .
$$

In this case, the canonical moments are given by [27]

$$
\mu_{n}^{(\alpha, \beta)}=\int_{0}^{1} z^{\alpha+n}(q z ; q)_{\beta} d_{q} z=\frac{(1-q)\left(q^{\alpha+\beta+1} ; q\right)_{\infty}(q ; q)_{\infty}\left(q^{\alpha+1} ; q\right)_{n}}{\left(1-q^{\alpha+\beta+1}\right)\left(q^{\alpha+1} ; q\right)_{\infty}\left(q^{\beta+1} ; q\right)_{\infty}\left(q^{\alpha+\beta+2} ; q\right)_{n}} .
$$

If we define monic little $q$-Jacobi polynomials $\left\{\tilde{p}_{n}^{(\alpha, \beta)}(z ; q)\right\}_{n \geq 0}$

$$
\tilde{p}_{n}^{(\alpha, \beta)}(z ; q)=(-1)^{n} q^{\frac{n(n-1)}{2}} \frac{\left(q^{\alpha+1} ; q\right)_{n}}{\left(q^{\alpha+\beta+n+1} ; q\right)_{n}} p_{n}^{(\alpha, \beta)}(z ; q)
$$


then they satisfy the orthogonal relation

$$
\begin{aligned}
& \int_{0}^{1} \tilde{p}_{n}^{(\alpha, \beta)}(z ; q) \tilde{p}_{m}^{(\alpha, \beta)}(z ; q) z^{\alpha}(q z ; q)_{\beta} d_{q} z \\
& \quad=q^{n(n+\alpha)}[\alpha+\beta+2 n+1]_{q}^{-1} \frac{(q ; q)_{n}(q ; q)_{n+\alpha}(q ; q)_{n+\beta}(q ; q)_{n+\alpha+\beta}}{(q ; q)_{2 n+\alpha+\beta}^{2}} \delta_{n, m}:=h_{n} \delta_{n, m} .
\end{aligned}
$$

Regarding the weight of little $q$-Jacobi polynomials

$$
\rho(z ; q)=z^{\alpha}(q z ; q)_{\beta}
$$

by solving (3.3), it admits the Pearson pair

$$
(f, g)=\left(-x^{2}+x,-q^{\frac{1}{2}}\left([\alpha+\beta+2]_{q} x-[\alpha+1]_{q}\right)\right) \text {. }
$$

Therefore, the coefficient $c_{n}$ has the expression

$$
c_{n}=q^{-n}[2 n+2+\alpha+\beta]_{q} h_{n+1},
$$

where $h_{n}$ is the nomalization constant in the orthogonal relation of monic polynomials (3.9). Then we have the following expression for the moment Pfaffian

$$
\begin{aligned}
\operatorname{Pf}\left(m_{i j}^{(\alpha, \beta)}\right)_{i, j=0}^{2 N-1} & =q^{\frac{1}{3} N\left(4 N^{2}-3 N+2\right)+\alpha N^{2}} \\
& \times \prod_{k=0}^{N-1} \frac{\left(1-q^{\alpha+\beta+4 k+2}\right)\left(q, q^{\alpha+1}, q^{\beta+1}, q^{\alpha+\beta+1} ; q\right)_{2 k+1}(q ; q)_{\infty}\left(q^{\alpha+\beta+1} ; q\right)_{\infty}}{\left(q^{\alpha+1}, q^{\beta+1} ; q\right)_{\infty}\left(q^{\alpha+\beta+1} ; q\right)_{4 k+2}\left(q^{\alpha+\beta+1} ; q\right)_{4 k+3}}
\end{aligned}
$$

Since the weight of the corresponding skew orthogonal little $q$-Jacobi polynomials is

$$
\omega(x ; q)=f(q x ; q) \rho^{(\alpha, \beta)}(q x ; q)=q^{\alpha+1} \rho^{(\alpha+1, \beta+1)}(x ; q)=q^{\alpha+1} x^{\alpha+1}(q x ; q)_{\beta+1},
$$

the skew moments $\left\{m_{i, j}^{(\alpha, \beta)}\right\}_{i, j \in \mathbb{N}}$ are related to the canonical moments by

$$
\begin{aligned}
m_{i, j}^{(\alpha, \beta)} & =\left([j]_{q}-[i]_{q}\right) \int_{0}^{1} x^{i+j-1} \omega(x ; q) d_{q} x=\left([j]_{q}-[i]_{q}\right) q^{\alpha+1} \mu_{i+j-1}^{(\alpha+1, \beta+1)} \\
& =\left([j]_{q}-[i]_{q}\right) q^{\alpha+1} \frac{(1-q)\left(q^{\alpha+\beta+1} ; q\right)_{\infty}(q ; q)_{\infty}\left(q^{\alpha+1} ; q\right)_{n}}{\left(1-q^{\alpha+\beta+1}\right)\left(q^{\alpha+1} ; q\right)_{\infty}\left(q^{\beta+1} ; q\right)_{\infty}\left(q^{\alpha+\beta+2} ; q\right)_{n}}
\end{aligned}
$$

After eliminating the constant $\left[q^{\alpha+1}\left(q^{\alpha+\beta+1} ; q\right)_{\infty}(q ; q)_{\infty} /\left(\left(1-q^{\alpha+\beta+1}\right)\left(q^{\alpha+1}, q^{\beta+1} ; q\right)_{\infty}\right)\right]^{N}$, we have

$$
\begin{aligned}
& \operatorname{Pf}\left(\left([j]_{q}-[i]_{q}\right) \frac{(1-q)\left(q^{\alpha+2} ; q\right)_{i+j-1}}{\left(q^{\alpha+\beta+4} ; q\right)_{i+j-1}}\right)_{i, j=0}^{2 N-1} \\
& \quad=q^{\frac{1}{3} N(N-1)(4 N+1)+\alpha N(N-1)} \prod_{k=0}^{N-1} \frac{(q ; q)_{2 k+1}\left(q^{\alpha+2}, q^{\beta+2} ; q\right)_{2 k}}{\left(q^{\alpha+\beta+4} ; q\right)_{2 k-2}\left(q^{\alpha+\beta+2 k+2} ; q\right)_{2 k}\left(q^{\alpha+\beta+2 k+2} ; q\right)_{2 k+2}}
\end{aligned}
$$

which coincides with the result in [20, Thm. 5.1]. 
3.4. Big $q$-Jacobi case. Big $q$-Jacobi polynomials were introduced by Andrews and Askey as an infinite-dimentional version of the $q$-Hahn polynomials [4]. In addition, big $q$-Jacobi polynomials were also contained in the Bannai-Ito scheme of dual systems of orthogonal polynomials as an infinite dimension analogue of the q-Racah polynomials [6]. These polynomials take the hypergeometric function form

$$
P_{n}(x ; a, b, c ; q)={ }_{3} \phi_{2}\left(\begin{array}{c|c}
q^{-n}, a b q^{n+1}, x \\
a q, c q
\end{array} \mid q ; q\right),
$$

orthogonal with respect to the weight

and have the orthogonality

$$
\rho^{(a, b, c)}(x ; q)=\frac{\left(a^{-1} x, c^{-1} x ; q\right)_{\infty}}{\left(x, b c^{-1} x ; q\right)_{\infty}}
$$

$$
\begin{aligned}
& \int_{c q}^{a q} P_{m}(x ; a, b, c ; q) P_{n}(x ; a, b, c ; q) \rho^{(a, b, c)}(x ; q) d_{q} x \\
& \quad=a q(1-q) \frac{\left(q, a^{-1} c, a c^{-1} q, a b q^{2} ; q\right)_{\infty}}{\left(a q, b q, c q, a b c^{-1} q ; q\right)_{\infty}} \frac{(1-a b q)}{\left(1-a b q^{2 n+1}\right)} \frac{\left(q, b q, a b c^{-1} q ; q\right)_{n}}{(a b q, a q, c q ; q)_{n}}\left(-a c q^{2}\right)^{n} q^{\left(\begin{array}{c}
n \\
2
\end{array}\right)} \delta_{m n} .
\end{aligned}
$$

One can easily check that the normalisation constant for the monic polynomials is

$$
h_{n}=\frac{a q(1-q)\left(-a c q^{2}\right)^{n} q^{\left(\begin{array}{c}
n \\
2
\end{array}\right)}\left(q, a^{-1} c, a c^{-1} q, a b q ; q\right)_{\infty}\left(q, a q, b q, c q, a b c^{-1} q ; q\right)_{n}}{\left(1-a b q^{2 n+1}\right)\left(a q, b q, c q, a b c^{-1} q ; q\right)_{\infty}(a b q ; q)_{n}\left(a b q^{n+1} ; q\right)_{n}^{2}}
$$

and the canonical moments are given by [27]

$$
\begin{aligned}
\mu_{n}^{(a, b, c)} & =\int_{c q}^{a q} x^{n} \rho^{(a, b, c)}(x ; q) d_{q} x \\
& =a q \frac{\left(a b q^{2}, a^{-1} c, a c^{-1} q ; q\right)_{\infty}}{\left(a q, b q, c q, a b c^{-1} q ; q\right)_{\infty}} \sum_{m=0}^{n}(-1)^{m}\left[\begin{array}{c}
n \\
m
\end{array}\right]_{q} q^{-n m+\left(\begin{array}{c}
m+1 \\
2
\end{array}\right)} \frac{(a q, c q ; q)_{m}}{\left(a b q^{2} ; q\right)_{m}} .
\end{aligned}
$$

Solving equation (3.3) gives the Pearson pair

$$
(f, g)=\left(\left(1-\frac{x}{a q}\right)\left(1-\frac{x}{c q}\right), \frac{q^{\frac{1}{2}}}{1-q}\left(\left(\frac{1}{a c q^{2}}-\frac{b}{c}\right) x+\frac{b}{c}+1-\frac{1}{a q}-\frac{1}{c q}\right)\right) .
$$

Then, by comparing the leading coefficients on both sides of (3.8) we have

$$
c_{n}=\frac{a b q^{2 n+2}-1}{a c(1-q) q^{n+2}} h_{n+1},
$$

which leads to the following evaluation of the moment Pfaffian

$$
\begin{aligned}
\operatorname{Pf}\left(m_{i j}^{(a, b, c)}\right)_{i, j=0}^{2 N-1} & =(-1)^{N} c^{N(N-1)} a^{N^{2}} q^{\frac{1}{6} N(N+1)(4 N-1)} \\
& \times \prod_{k=0}^{N-1} \frac{\left(q, a^{-1} c, a c^{-1} q, a b q ; q\right)_{\infty}\left(q, a q, b q, c q, a b c^{-1} q ; q\right)_{2 k+1}}{\left(a q, b q, c q, a b c^{-1} q ; q\right)_{\infty}(a b q ; q)_{2(k+N)+1}} .
\end{aligned}
$$

where $m_{i, j}^{(a, b, c)}$ is given by

$$
m_{i, j}^{(a, b, c)}=\left([j]_{q}-[i]_{q}\right) \int_{c q}^{a q} x^{i+j-1} \omega(x ; q)^{(a, b, c)} d_{q} x,
$$

and $\omega(x ; q)$ is expressed by

$$
\omega(x ; q)^{(a, b, c)}=f(q x ; q) \rho^{(a, b, c)}(q x ; q)=(1-x)\left(1-b c^{-1} x\right) \rho(x ; q)^{(a, b, c)}=\rho^{(q a, q b, q c)}(q x ; q) .
$$


Note that

$$
\mu_{n}^{(a, b, c)}=\int_{c q}^{a q} x^{n} \rho^{(a, b, c)}(x ; q) d_{q} x=q^{n+1} \int_{c}^{a} x^{n} \rho^{(a, b, c)}(q x ; q) d_{q} x
$$

we have the expression

$$
\begin{aligned}
m_{i, j}^{(a, b, c)} & =\left([j]_{q}-[i]_{q}\right) q^{-(i+j)} \mu_{i+j-1}^{(q a, q b, q c)} \\
& =\left([j]_{q}-[i]_{q}\right) a q^{-(i+j-1)} \frac{\left(a b q^{2}, a^{-1} c, a c^{-1} q ; q\right)_{\infty}}{\left(a q, b q, c q, a b c^{-1} q ; q\right)_{\infty}}\left(\sum_{m=0}^{n}(-1)^{m}\left[\begin{array}{c}
n \\
m
\end{array}\right]_{q} q^{-n m+\left(\begin{array}{c}
m+1 \\
2
\end{array}\right)} \frac{(a q, c q ; q)_{m}}{\left(a b q^{2} ; q\right)_{m}}\right)
\end{aligned}
$$

Dividing both sides by $\left[a q\left(a b q^{2}, a^{-1} c, a c^{-1} q ; q\right)_{\infty} /\left(a q, b q, c q, a b c^{-1} q ; q\right)_{\infty}\right]^{N}$ and changing variables $(a, b, c) \rightarrow\left(q^{-1} a, q^{-1} b, q^{-1} c\right)$, we get

$$
\begin{aligned}
& \operatorname{Pf}\left(\left([j]_{q}-[i]_{q}\right) \sum_{m=0}^{i+j-1}(-1)^{m}\left[\begin{array}{c}
i+j-1 \\
m
\end{array}\right]_{q} q^{-(i+j-1)(m+1)+\left(\begin{array}{c}
m+1 \\
2
\end{array}\right)} \frac{(a q, c q ; q)_{m}}{\left(a b q^{2} ; q\right)_{m}}\right)_{i, j=0}^{2 N-1} \\
& \quad=(-1)^{N}(a c)^{N(N-1)} q^{\frac{1}{6} N\left(4 N^{2}-9 N+11\right)} \prod_{k=0}^{N-1} \frac{(q ; q)_{\infty}(q ; q)_{2 k+1}\left(a, b, c, a b c^{-1} q ; q\right)_{2 k}}{\left(a b q^{2} ; q\right)_{2(k+N)-2}} .
\end{aligned}
$$

Remark 3.2. With $a=b=1$, big $q$-Jacobi polynomials reduce to the big q-Legendre polynomials [23]. In particular, we have the following evaluation of q-Catalan-Hankel Pfaffian

$$
\begin{aligned}
& \operatorname{Pf}\left(\left([j]_{q}-[i]_{q}\right) \sum_{m=0}^{i+j-1}(-1)^{m}\left[\begin{array}{c}
i+j-1 \\
m
\end{array}\right]_{q} q^{-(i+j-1)(m+1)+\left(\begin{array}{c}
m+1 \\
2
\end{array}\right)} \frac{(q, c q ; q)_{m}}{\left(q^{2} ; q\right)_{m}}\right)_{i, j=0}^{2 N-1} \\
& =(-1)^{N}(c)^{N(N-1)} q^{\frac{1}{6} N\left(4 N^{2}-9 N+11\right)} \prod_{k=0}^{N-1} \frac{(q ; q)_{\infty}(q ; q)_{2 k+1}\left(q, q, c q, c^{-1} q ; q\right)_{2 k}}{\left(q^{2} ; q\right)_{2(k+N)-2}} .
\end{aligned}
$$

\section{FURTHER REMARKS}

In this paper, we have developed a method based on the relation between classical ( $q$-)orthogonal and $(q$-)skew orthogonal polynomials to evaluate certain $q$-Catalan-Hankel Pfaffians whose entries are composed of the moments of classical orthogonal polynomials. Some examples are given to illustrate the approach including the continuous ones (e.g. Hermite, Laguerre, Jacobi and Cauchy) and discrete $q$-cases (e.g. Al-Salam \& Carlitz I, little $q$-Jacobi, Stieltjes-Wigert and big $q$-Jacobi polynomials). Amoung those examples, the Al-Salam \& Carlitz I and Little q-Jacobi case are compared with the results obtained by M. Ishikawa and J. Zeng in [20] and we present alternative proofs of [20, eq. (6.7) \& Thm 5.2]. Besides, the examples in [20, Conjecture 7.1] seem to be related to some discrete measure on the linear lattice. However, as mentioned in [16], the skew moments defined in linear lattices are of the form $\mu_{i, j}=(j-i) \mu_{i+j-1}+\left(\left(\begin{array}{l}j \\ 2\end{array}\right)-\left(\begin{array}{l}i \\ 2\end{array}\right)\right) \mu_{i+j-2}+\cdots$, it is unclear to us whether it could be written in the Catalan-Hankel Pfaffian form. 
As mentioned before, $q$-analogues of Selberg integral can be used to evaluate $q$-Catalan-Hankel Pfaffians. For example, the formula (3.10) can be evaluated by the following Askey-HabsiegerKadell formula [20]

$$
\begin{aligned}
& \int_{[0,1]^{n}} \prod_{i<j} t_{i}^{2 k}\left(q^{1-k} t_{j} / t_{i} ; q\right)_{2 k} \prod_{i=1}^{n} t_{i}^{x-1} \frac{\left(t_{i} q ; q\right)_{\infty}}{\left(t_{i} q^{y} ; q\right)_{\infty}} d_{q} t \\
& \quad=q^{k x\left(\begin{array}{l}
n \\
2
\end{array}\right)+2 k^{2}\left(\begin{array}{c}
n \\
3
\end{array}\right)} \prod_{j=1}^{n} \frac{\Gamma_{q}(x+(j-1) k) \Gamma_{q}(y+(j-1) k) \Gamma_{q}(j k+1)}{\Gamma_{q}(x+y+(n+j-2) k) \Gamma_{q}(k+1)} .
\end{aligned}
$$

Conversely, in this paper, we give an explanation to this integral identity in terms of the skew little $q$-Jacobi polynomials. It is still an open question to construct classical skew orthogonal polynomials to make explanations of some other discrete Selberg integrals such as [7, Prop. 5.1]

$$
\begin{aligned}
& \sum_{k_{1}, \cdots, k_{r}=-n}^{n} \prod_{1 \leq i<j \leq r}\left|\left[k_{i}-k_{j}\right]_{q}\right| \prod_{i=1}^{r} q^{\left(k_{i}+n-r+i\right)^{2} / 2}\left[\begin{array}{c}
2 n \\
n+k_{i}
\end{array}\right] \\
& =\frac{r !}{|r|_{q^{1 / 2}} !} \prod_{i=1}^{r}\left(-q^{1 / 2} ; q^{1 / 2}\right)_{i}\left(-q^{i / 2+1} ; q\right)_{2 n-r} \prod_{i=1}^{r} \frac{\Gamma_{q}(1+i / 2)}{\Gamma_{q}(3 / 2)} \frac{\Gamma_{q}(2 n+1) \Gamma_{q}(2 n-i+5 / 2)}{\Gamma_{q}(2 n-i+2) \Gamma_{q}(2 n-i / 2+2)} .
\end{aligned}
$$

Moreover, combinatoric explanations of $q$-Catalan-Hankel Pfaffians are left to be further investigated.

\section{References}

[1] M. Adler, P. Forrester, T. Nagao and P. van Moerbeke. Classical skew orthogonal polynomials and random matrices. J. Stat. Phys. 99 (2000), 141-170.

[2] M. Adler, E. Horozov and P. van Moerbeke. The Pfaff lattice and skew-orthogonal polynomials. Internat. Math. Res. Notices 1999, 569-588.

[3] G. Andrews, R. Askey and R. Roy. Special functions. Encyclopedia Math. Appl., vol. 71, Cambridge University Press, Cambridge, 2000.

[4] G.E. Andrews and R. Askey. Classical orthogonal polynomials. Polynômes Orthogonaux et Applications, Lecture Notes in Mathematics, 1985, V. 1171, 36-62. MR838970 (88c:33015b)

[5] R. Bacher. Determinants of matrices related to the Pascal triangle. J. Théor. Nombres Bordeaux, 14 (2002), 19-41.

[6] E. Bannai and T. Ito. Algebraic Combinatorics I: Association Schemes. Benjamin \& Cummings, Menlo Park, CA, 1984. MR882540 (87m:05001)

[7] R. Brent, C. Krattenthaler and O. Warnaar. Discrete analogues of Macdonald-Mehta integrals. J. Comb. Theory Ser. A, 144 (2016), 80-126.

[8] X. Chang, Y. He, X. Hu and S. Li. Partial-skew-orthogonal polynomials and related integrable lattices with Pfaffian tau-functions. Commun. Math. Phys., 364 (2018), 1069-1119.

[9] T. Chihara. A characterization and a class of distribution functions for the Stieltjes-Wigert polynomials. Canad. Math. Bull., 13 (1970), 529-532.

[10] T.S. Chihara. An Introduction to Orthogonal Polynomials. Mathematics and its Applications, vol. 13, Gordon and Breach, 1978.

[11] J. Christiansen. The moment problem associated with the Stieltjes-Wigert polynomials. J. Math. Anal. Appl., 277 (2003), 218-245.

[12] G. Han. Hankel continued fraction and its applications. Adv. Math., 303 (2016), 295-321.

[13] P. Forrester. Log-gases and random matrices. London Mathematical Society Monographs Series, Vol. 34, Princeton University Press, Princeton, NJ, 2010. 
[14] P. Forrester. Meet Andréief, Bordeaux 1886, and Andreev, Kharkov 1882-1883. Random Matrices Theory Appl. 8 (2019), 1930001, 9 pp.

[15] P. Forrester. Global and local scaling limit for the $\beta=2$ Stieltjes-Wigert random matrix ensemble. arXiv: 2011.11783.

[16] P. Forrester and S. Li. Classical discrete symplectic ensembles on the linear and exponential lattice: skew orthogonal polynomials and correlation functions. Trans. Amer. Math. Soc., 373 (2020), 665-698.

[17] P. Forrester and S. Warnaar. The importance of the Selberg integral. Bull. Amer. Math. Soc., 45 (2008), 489-534.

[18] G.H. Hardy and E.M. Wright. An Introduction to The Theory of Numbers. 4th ed., Oxford University Press, Oxford, 1960.

[19] M. Ishikawa, H. Tagawa and J. Zeng. Pfaffian decomposition and a Pfaffian analogue of q-Catalan Hankel determinants. J. Comb. Theory. A, 120 (2013) 1263-1284.

[20] M. Ishikawa and J. Zeng. Hankel hyperpfaffian calculations and Selberg integrals. arXiv: 2008.09776v1.

[21] M. Ismail. Classical and quantum orthogonal polynomials in one variable, with two chapters by W. van Assche. Encyclopedia of Mathematics and its Applications, Vol. 98, Cambridge University Press, Cambridge, England, 2005.

[22] R. Koekoek, P. Lesky and R. Swarttouw. Hypergeometric orthogonal polynomials and their qanalogues. Springer Monographs in Mathematics, Springer-Verlag Berlin Heidelberg 2010.

[23] S. Li and G. Yu. Christoffel transformations for (partial-)skew-orthogonal polynomials and applications. arXiv: 2008.00273, 2020.

[24] T. Masuda, K. Mimachi, Y. Kakagami, M. Noumi and K. Ueno. Representations of the quantum group $S U_{q}(2)$ and the little q-Jacobi polynomials. J. Func. Anal., 99 (1991), 357-386.

[25] M. Mehta and R. Wang. Calculation of a certain determinant. Commun. Math. Phys., 214 (2000) $227-232$.

[26] A. Nikiforov and S. Suslov. Classical orthogonal polynomials of a discrete variable on nonuniform lattices. Lett. Math. Phys., 11 (1986), 27-34.

[27] P. N. Sadjang, W. Koepf and M. Foupouagnigni. On moments of classical orthogonal polynomials. J. Math. Anal. Appl. 424 (2015) 122-151.

[28] T.J. Stieltjes. Recherches sur les fractions continues. Ann. Fac. Sci. Toulouse 8 (1894) 1-122; Ann. Fac. Sci. Toulouse 9 (1895) 1-47; English version contained in T.J. Stieltjes, Collected Papers, G. van Dijk (Ed.), Vol. II, Springer, Berlin, 1993.

[29] R. Vein and P. Dale. Determinants and their applications in mathematical physics. Applied Mathematical Sciences, Vol 134, Springer-Verlag, New York, 1999.

[30] S. Wigert. Sur les polynômes orthogonaux et l'approximation des fonctions continues. Arkiv för matematik, astronomi och fysik 17 (18) (1923).

School of Mathematical Sciences, Shanghai Jiaotong University, People's Republic of China.

Email address: JOHN-EINSTEIN@sjtu.edu.cn

Department of Mathematics, Sichuan University, Chengdu, 610064, China

Email address: lishihao@lsec.cc.ac.cn

School of Mathematical Sciences, Shanghai Jiaotong University, People's Republic of China.

Email address: gfyu@sjtu.edu.cn 